\section{ABNORMAL PROTEODERMATAN SULFATE IN THREE
PATIENTS WITH COFFIN-LOWRY SYNDROME}

Michael Beck, Josef Glössl, Rainer Rüter, and Hans Kresse

Institute of Physiological Chemistry,

University of Münster, D-4400 Münster,
Federal Republic of Germany

SUMMARY

The properties of $\left[^{35}\right.$ s] sulfate-labeled, proteoglycans secreted by normal human skin fibroblasts were compared with those synthesized by fibroblasts from three patients with Coffin-lowry
syndrome. 60-808 of secreted radioactive macromolecules from syndrome. $60-808$ of secreted radioactive macromolecules from a mean $\mathrm{Kav}_{\mathrm{av}}$-value of 0.56 (pool 2); 3-10\% of the radioactivit appeared in the exclusion volume of the column (pool 1). In contrast, 17-60\% of the proteoglycans from the patients were found in the vold volume. The bulk of remaining material was eluted with a mean $\mathrm{Kav}_{\mathrm{aval}} \mathrm{ve}$ of 0.47 . Pool 2 glycan cha
exhibited an increased hydrodynamic size.

pool 1 from normal cells contained predominantly a glucuronic acid-rich proteodermatan sulfate, iduronic acid amounting for approximately $20 \%$ of glucuronic acid. In the respective proteodermatan sulfate from the patients, the relative iduronic acid content was at least 338 of that of glucuronic acid. Pool 2 material of all cell lines was characterized predominantly as iduronic acid-rich proteodermatan sulfate. In the proteoglycans disaccharides was increased at the expense of 6 -sulfated chondroitin disaccharides. Native proteoglycans from the patients were less efficiently endocytosed by fibroblasts than their normal counterparts.

Coffin-Lowry fibroblasts had a normal capability to synthesize glycosaminoglycan chains on an arteficial acceptor, p-nitrophenyl chondroitin 4 - and 6 -sulfotransferase activities.

\section{INTRODUCTION}

The Coffin-Lowry syndrome is a rare genetically transmitted disorder that is marked by mental retardation, thick, soft skin, tapering fingers, a characteristic facies and skeletal abnormalities $(5,8,9,12)$. Males are more severely affected than
females, suggesting $\mathrm{x}$-linked semi-dominant transmission.

The primary defect of the Coffin-Lowry syndrome is completely unknown. Coffin et al. (5) found a drastically reduced number of elastic fibers in the skin of one patient, but this observation could not be confirmed in other cases. Temtamy et al. (12) considered the appearance of inclusion bodies with fine fibrillogranular content as pathognomonic, but the quantity of membraneTo our knowledge, no search for the accumulation of other

When we first were confronted with a patient with coffin-Lowry syndrome, we considered the possibility that the appearance of
the skin could be caused by an excessive extracellular accumulatthe skin could be caused by an excessive extracellular accumulatproteoglycans reenter the cells by receptor-mediated endocytosis before complete degradation within the lysosomes takes place. In case of proteodermatan sulfate the recognition marker required for this uptake process resides in its protein moiety (13), so result in its extracellular accumulation.

Proteodermatan sulfate is of greatest quantitative importance among the proteoglycans secreted by skin fibroblasts. Proteodermatan sulfate from embryonic human skin fibroblasts could be separated into two types, a large proteoglycan with glucuroni acid-rich and a smaller proteoglycan with iduronic acid-rich structure of cell-associated and secreted dermatan sulfate have structure of cell-associated
been described $(3,4,11)$.

We will report here that structural and functional anomalies

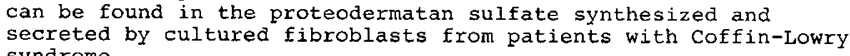
syndrome.

\section{PATIENTS}

Patient 1 (J.B.) is a $19-y-o l d$ boy who was diagnosed by Dr. U. Feldmann of this university. The patient exhibits the full clinical picture of the Coffin-Lowry syndrome and especially suffers from spine deformities. The mother and two sisters of the patient showed no obvious sign of the disease. Patient 2 (C.S.) is a 4-y-old boy, also diagnosed by Dr. Feldmann. He has an affected eldex brother, and his mothex seems to be mildly affected. These patients will be described in detail elsewhere. From patient clinical data were not available to us.

\section{METHODS}

Fibroblast cultures from the patients and from normal individuals (age $3-30$ y) were obtained from skin biopsies and further propagated in modified Eagle's Minimum Essential Medium two different occasions. The cultures were used between the fourth and sixteenth passage. In each set of were used between the four patients and controls had undergone a similar number of subcultures. There was no obvious difference in the growth characteristics of the fibroblasts from the patients and controls.

$\left[{ }^{35} \mathrm{~S}_{0} \mathrm{O}_{4}\right.$-labeled proteoglycans were prepared in the following manner: Fibroblasts were grown to confluency in a $75 \mathrm{~cm}^{2}$ Falcon plastic flask. The labeling medium, $10 \mathrm{ml}$, was the same medium but deficient in streptomycin sesquisulfate and supplemented with
$150 \mu \mathrm{Ci} \mathrm{Na}_{2}\left(35 \mathrm{~S}^{\circ} \mathrm{O}_{4}\right.$ (carrier-free); Amersham-Buchler, Braunschweig, Germany). In some experiments, $\mathrm{Na}_{2}\left({ }^{35} \mathrm{~S}\right) \mathrm{O}_{4}$ was replaced by $150 \mu \mathrm{Ci}$ $\left[6-3_{\mathrm{H}}\right] \mathrm{glucosamine}$ hydrochloride (sp. radioactivity $38 \mathrm{Ci} / \mathrm{mmole}$;
Amersham-Buchler); in others, both isotopes were used. At the end of the incubation period, usually after $48 \mathrm{~h}$, medium was made 708 saturated with $\left(\mathrm{NH}_{2}\right)_{2} \mathrm{SO}_{4}$. The precipitate was collected by centrifugation and dissolved in the original volume of $20 \mathrm{mM}$ Tris/HCl buffer, $\mathrm{pH} 7.5$, containing $0.1 \mathrm{M}$ 6-aminohexanoic acid, $10 \mathrm{mM}$ EDTA, $5 \mathrm{mM}$ benzamidine, $10 \mathrm{mM}$ N-ethylmaleimide, $0.18(\mathrm{v} / \mathrm{v})$ Triton $\mathrm{X}-100$ (buffer A), and $0.15 \mathrm{M}$ NaCl. After dialysis overnight against this buffer, the retentate was loaded on a $0.7 \times 13 \mathrm{~cm}$ DEAETrisacryl (LKB Instruments, Gräfelfing, Germany) column, equilibrated with this buffer. The column was eluted stepwise with $15 \mathrm{ml}$ each of $0.15 \mathrm{M} \mathrm{NaCl}, 0.3 \mathrm{NaCl}$, and $1.0 \mathrm{M} \mathrm{NaCl}$, all in buffer $\mathrm{A}$. Appropriate fractions from the last step were pooled and dialyzed against 0.18 Triton $x-100$. The detergent was used to minimize ware and dialysis bags.

For gel chromatography the samples were dried under reduced pressure and then dissolved in $50 \mathrm{mM}$ sodium acetate buffer, pH 6.0, containing $4 \mathrm{M}$ guanidinium hydrochloride and the protease
inhibitor cocktail mentioned above. The material was loaded onto a $1.2 \times 100 \mathrm{~cm}$ Sepharose CL-4B (Pharmacia, Freiburg, Germany) column equilibrated with the same buffer, and chromatographed at a flow rate of $6 \mathrm{ml} / \mathrm{h}$, fraction volume $1.0 \mathrm{ml}$. Appropriate fractions were pooled, dialyzed against $0.1 \%$ Triton $\mathrm{X}-100$, and concentrated in vacuo. Liberation of glycosaminoglycan chains by 8 -elimination was done as described (13). The samples were concentrated again, salt was removed by washing with methanol, and the samples were digested in parallel with $20 \mathrm{mU}$ chondroitin AC lyase (EC 4.2.2.5; Sigma, Munich, Germany) and $20 \mathrm{mu}$ chondroitin ABC lyase (EC 4.2.2.4; Sigma), respectively, for $4 \mathrm{~h}$ at $370^{\circ} \mathrm{C}$, final volume $20 \mu 1$ (15). The digest was spotted on Schleicher and Schüll paper 2043b (Dassel, Germany) and subjected to descending paper chromatography in butan-1-ol/1 $\mathrm{M} \mathrm{NH}_{3}$ /acetic acid $12: 1: 3$, in scintillation vials with $1 \mathrm{ml} \mathrm{H}_{2} \mathrm{O}$ before liquid scintillation in scintillation vials with $1 \mathrm{ml} \mathrm{H}_{2} \mathrm{O}$ before liquid scintillation proportion of radioactivity that was resistant towards chondroitin $A B C$ lyase. The amount of dermatan sulfate was obtained from the difference of radioactivity degraded by
chondroitin $\mathrm{AC}$ lyase and chondroitin $\mathrm{ABC}$ lyase.

For treatment of proteoglycans with hyaluronate lyase (EC 4.2.2.1) (Calbiochem-Behring, Giessen, Germany), Sepharose CL-4B fractions were concentrated after dialysis, Triton $X-100$
was removed by washing with methanol, and the sample was dissolved in $120 \mu 150 \mathrm{~mm}$ sodium phosphate buffer, $\mathrm{pH} 6.0$, containing 100 turbidity reducing units of enzyme, $0.33 \mathrm{mM}$ pepstatin, and the was rechromatographed under dissociative conditions.

The hydrodynamic size of glycosaminoglycan chains was determined by chromatography on a $1.2 \times 90 \mathrm{~cm}$ Sephacryl $\mathrm{s}-300$ (Pharmacia) column which had been equilibrated with $50 \mathrm{mM}$ sodium acetate buffer, $\mathrm{pH} 6.0$, containing $4 \mathrm{M}$ guanidinium chloride. The provided by Dr. Wasteson, Uppsala, Sweden $(14)$.

Studies on the metabolism of exogenously added $\left[{ }^{35} \mathrm{SjO}_{4}\right.$-labeled proteoglycans were performed as described (13), except that the as mentioned above. Triton $x-100$ was omiaphy on DEAE-Trisacry as mentioned above. Triton $X-100$ was omitted from all buffers. or absence of p-nitrophenyl-B-D-xyloside were done as quoted

Chondroitin sulfotransferase activity (EC 2.8.2.?) was determined in the following manner: $100 \mathrm{\mu g}$ chondroitin (kindly provided by $D$. Göhler of this institute), approx. $150 \mathrm{\mu g}$ of cell activity $50 \mathrm{Ci} / \mathrm{mole}$, prepared as in 10 ) were incubated for $24 \mathrm{~h}$ at $22^{\circ} \mathrm{C}$ in $50 \mathrm{mM}$ sodium phsophate buffer, $\mathrm{pH} 7.0$, containing $1 \mathrm{mM}$ $\mathrm{MgCl}_{2}, 1 \mathrm{mM} \mathrm{MnCl} 2,10 \mathrm{mM} \mathrm{KF}, 80 \mathrm{mM} \mathrm{NaCl}, 0.02 \% \mathrm{NaN}_{3}$ and $0.05 \%$ Triton $\mathrm{x}-100$, final volume $105 \mu 1$. Sulfated product was recovered by high pressure liquid chromatography on a TSK $G 2000$ SW column
(Varian, Darmstadt, Germany) before dialysis and degradation with
chondroitin ABC lyase. chondroitin $A B C$ lyase.

\section{RESULTS}

After biosynthesis, proteoglycans are distributed into different compartments. In case of cultured skin fibroblasts, the bulk of material is secreted into the culture medium. A minor part remains inside the cell and is preferentially transferred to regard to the rate of biosynthesis of proteoglycans and their distribution into these pools (Fig 1). The turnover of intracellular glycosaminoglycans is also normal (result not shown). Addition

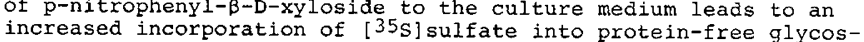
increased incorporation of $[35 \mathrm{~S}]$ sulfate into protein-free glycosaminoglycan chains. Glycosaminoglycan biosynthesis of Coffin-Lowry cells (Fig 1).

Gel chromatography of secreted proteoglycans from the patients, however, revealed an abnormality of their hydrodynamic size. As shown in Fig $2 \mathrm{D}, 3-10 \%(\mathrm{n}=5)$ of radioactive proteoglycans from normal cells (age of the donors was $3-12$ y) were eluted in the exclusion volume of a Sepharose $\mathrm{CL}-4 \mathrm{~B}$ column when the column was be referred to as pool 1 material. In the patients, 17-60\% of the label were found in the void volume (Fig $2 \mathrm{~A}-\mathrm{C}$ ). It should be noted that there was considerable variability even within one cell line. The proportion of large size proteoglycans of patient 1 who was most intensively studied, varied between $30-60 \%$ in four sifferent skin biopsies were used.

The bulk of secreted proteoglycans from normal cells was eluted from the Sepharose CL-4B column with a mean Kav-value of patients represented larger material because its Kav-value 0.47 . The increased hydrodynamic size was ascertained by cochromatography of $[35$ s] sulfate-labeled proteoglycans from patient 1 , and control material obtained after incubation in the 
presence of [ $3 \mathrm{H}]$ glucosamine ( $\mathrm{Fig} 2 \mathrm{~A}$ ). Differences in the elution profiles of $[35 \mathrm{~S}]-$ and $[3 \mathrm{H}]-$ labeled material are mainly due to labeling of hyaluronate in the latter case.

Excessive amounts of pool 1 proteoglycans were most likely not generated by the formation of abnormally stable aggregates of and $[3 \mathrm{H}]-$ labeled pool 1 proteoglycans from patient 1 with hyaluronate lyase and rechromatography on Sepharose CL-4B, no pool 2 proteoglycans could be recovered though the molecular size was diminished (Fig 3). It could not be decided whether this was due to proteolytic degradation by a non-inhibitable protease, or

An increased size of the patients'proteoglycans from pool 2 could be caused by an increased number of glycan chains and/or an increased chain length. Protein-free carbohydrate chains were obtained by B-elimination and chromatographed on a Sephacryl
S-300 column. Figure 4 shows that pool 2 proteoglycans from patients 1 and 2 contained glycan chains of larger size than those from controls. Differences in the chain length of pool 1 proteoglycans were not observed.

Glycosaminoglycan distribution pattern. The composition of glycosaminoglycan chains of the proteoglycans from pool 1 and 2 was analyzed after specific enzymatic degradation. As shown in Table 1, pool 1 from normal cells contained predominantly a glucuronic acid-rich proteodermatan sulfate. Iduronic acid amounted for approximately $20 \%$ of glucuronic acid. In the respective proteodermatan sulfate from the patients, the relative lduronic acid bulk of pool 2 proteoglycans was characterized as iduronic acidrich proteodermatan sulfate, regardless of the cell line tested. in proteodermatan sules chondroitin 4-sulfate disaccharides at the expense of 6-sulfated chondroitin disaccharides. Such an abnormality was not observed in the proteoglycans from patient 2 .

Chondroitin sulfotransferase activity. It is shown in Table 2 that ibroblasts from patients 1 and 2 did neither differ significant rom normal cells in their chondroitin sulfotransferase activit nor in the position of the sulfate ester linkages formed. Thus, found in native proteoglycans from both patients (see above) cound in native proteoglycans from both patients (see above) ferase is used as acceptor under in vitro conditions.

Endocytosis of proteoglycans. Proteoglycans from the patients showed also a metabolic abnormality because they were less efficiently endocytosed by fibroblasts than their normal counterpart. A typical example is given in Table 3. It is evident that CoffinLowry fibroblasts are able to internalize normal proteoglycans at a normal rate, whereas the rate of endocytosis of Coffin-Lowry Lowry fibroblasts were used as recipients.

DISCUSSION

Several qualitative and quantitative abnormalities of proteodermatan sulfate secreted by Coffin-Lowry fibroblasts have been found in the present investigation: (1) an increased proportion of the large glucuronic acid-rich proteodermatan sulfate at the expense of the smaller iduronic acid-rich proteodermatan sulfate (2) an increased proportion of the iduronic acid content of the large proteoglycan; (3) an increased size of the smaller proteodermatan sulfate; (4) a reduced capability of secreted proteoglycans for being endocytosed. Furthermore, in two of three patients an altered ratio of $4-$ and 6 -sulfated chondroitin
sulfate disaccharides within the glycosaminoglycan chains was found.

Carlstedt et al. (3) reported that the core protein of the large proteodermatan sulfate species secreted by embryonic skin fibroblasts has an $\mathrm{M}_{r}$ of over 400,000 , whereas that of the smallex species has an $M_{r}=47,000$. This could suggest that both core proteins are genetically distinct. In preliminary experiments we have not yet been able to detect a $M_{x} 400,000$ core protein in the secretions of normal or Coffin-Lowry fibroblasts. We cannot therefore answer the question whether or not Coffin-Lowry fibrobla
in embryonic cells.

Alternatively, pool 1 proteoglycans could represent aggregates of pool 2 proteoglycans. Proteodermatan sulfate shows the phenomenon of self-association and may form aggregates with degradation of this polymer by a specific enzyme does not result in the conversion of pool 1 proteoglycans into pool 2 proteoin the conversion of pool proteoglycans into pool 2 proteoseems unlikely because self-association is abolished in the presence of $4 \mathrm{M}$ guanidinium chloride $(6)$. On the other hand, interactions of proteoglycans with components not yet fully characterized may be resistant to such treatment (7). If pool 1 material from the patients would arise in part by the formation of abnormally stable aggregates of pool 2 proteoglycans, one could explain the increased iduronic acid content of pool 1 could explain the increased iduronic acid content of pool 1
proteoglycans. It could then be discussed whether aggregate
formation is caused by the production of an aggregating component or by the formation of abnormal proteodermatan sulfate.

During and after biosynthesis of the core proteins, a variety of posttranslational modifications occurs: synthesis, modification and sulfation of glycan chains, attachment of $\mathrm{N}-$ and $\mathrm{O}-$ glycosidically linked oligosaccharides and, most likely, proteolytic processing. It has not yet been established in case of proteoglycans as and to what extent the amino acid sequence of the
The three Coffin-Lowry cell lines investigated had some, but not all, abnormalities of proteodermatan sulfate in common. Because the disease is genetically lethal in case of males, the frequency of new mutations must be high, and several allelic mutations should exist. As we studied secondary consequences of an unknown primary defect, it is not surprising that a certain variability was found. It is our present hypothesis that the primary defect of the coffin-Lowry syndrome may concern expression, biosynthesis and/or posttranslational processing of a proteodermatan sulfate core protein. The functionally altered proteoglycan cannot be endocytosed at a normal rate, and therefore accumulates extracellularly. Studies are in progress to analyze proteodermatan sulfate core proteins by a more direc approach. Though the present results cannot be interpreted unequivocally, they may be of help in establishing a method for a biochemical diagnosis of the disorder.

\section{REFERENCES AND NOTES}

1. Aplin, J.D. and Hughes, R.C.: Complex carbohydrates of the interactions and biological roles. Biochem. Biophys. Acta, 694: 375 (1982).

2. Cantz, M., Kresse, H., Barton, R.W. and Neufeld, E.F.: Corrective factors for inborn errors of mucopolysaccharide metabolism. In: V. Ginsburg: Methods in Enzymology,
pp. 884-897 (Academic Press, New York, London, 1972).

3. Carlstedt, I., Cöster, L. and Malmström, A.: Isolation and characterization of dermatan sulphate and heparan sulphat proteoglycans from fibroblast culture. Biochem. J., 197: 217 (1981).

4. Cöstex, L., Carlstedt, I. and Malmström, A.: Isolation of ${ }^{35} \mathrm{~S}-$ and 3 H-labelled proteoglycans from cultures of hum

5. Coffin, G.S., Siris, E. and Wegienca, L.C.: Mental retardation with osteocartilaginous anomalies. Am. J. Dis. Child., 112: 205 (1966)

6. Fransson, L.- $\AA$. Cöster, L., Malmström, A. and Sheehan, J.K.: Self-association of scleral proteodermatan sulfate. J. Biol. Chem., 257: 6333 (1982)

7. Garner, J.A. and CuIp, L.A.: Aggregation competence of proteoglycans from the substratum adhesion sites of murine fibroblasts. Biochemistry, 20:7350 (1981).

8. Hunter, A.G.W., Partington, M.W., and Evans, J.A.: The CoffinLowry syndrome. Experience from four centres. Clin. Genet., 21: 321 (1982).

9. Lowry, B., Miller, J.R. and Fraser, F.C.: A new dominant gene mental ret
496 (1971)

10. Pennings, E.J.M., Vrielink, R. and van Kempen, G.M.J.: Kinetics and mechanism of the rat brain phenol sulphotransferase reaction. Biochem. J., 173: 299 (1978).

11. Sjöberg, I., carlstedt, I., Cöster, L., Malmström, A. and glycosaminoglycans in cultures of human fibroblasts. Biochem. J., 178: 257 (1979).

12. Temtamy, S.A., Miller, J.D., Dorst, J.P., Maumenee, I.H. , Salinas, C. Lacaissie, Y. and Kenyon, K.R.: The Coffin-Lowry syndrome. A simply inherited trait comprising mental retardation, facio-digital anomalies and skeletal involvement. Birth Defect:
No. $6: 133(1975)$.

13. Truppe, W. and Kresse, H.: Uptake of proteoglycans and sulfated glycosaminoglycans by cultured skin fibroblasts. Eur. J. Biochem., 85: 351 (1978).

14. Wasteson, \&.: A method for the determination of molecular weight dispersion in chondroitin sulphate on a microgram level. Biochim. Biophys. Acta, 177: 152 (1969).

15. Yamagata, T., Saito, H., Habuchi, O. and Suzuki, S.: Purification and properties of bacterial chondroitinases
and chondrosulfatases. J. Biol. Chem., 243: 1523 (1968).

6. Informed consent was obtained from all subjects in this study.

17. This work is dedicated to Professor E. Buddecke on the occasion of his 60 th birthday.

18. The authors are very much indebted to Dr. Ursula Feldmann, Institute of Human Genetics, University of Münster, who made us aware of the Coffin-Lowry syndrome and introduced to us two families with affected patients. Provision of fibroblast culture from patient 3 by Dr. J. Gehler, acknowledged.

19. Requests for reprints should be addressed to: Dr. Hans Kresse Physiologisch-Chemisches Institut der wWU D-4400 Münster/West-Germany

20. This research was supported by the Deutsche Forschungsgemeinschaft (SFB 104). M.B. is recipient of a training
fellowship from the Deutsche Forschungsgemeinschaft.

21. Received for publication March 15, 1983.

22. Accepted for publication July 13, 1983. 
1. Relative distribution pattern of [35S]glycosaminoglycans of pool 1 and pool 2

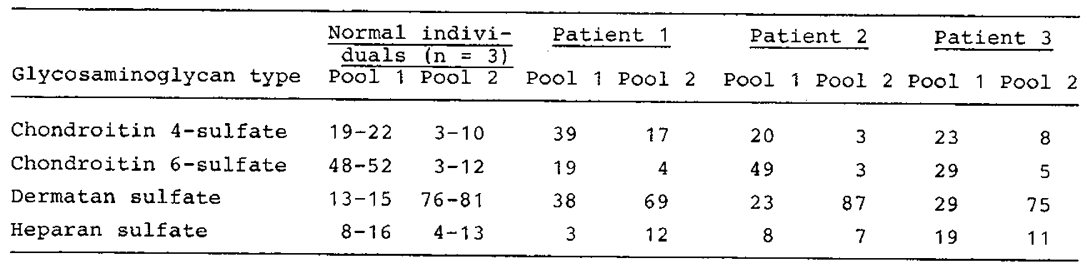

Pool 1 was obtained from the proteoglycans eluting in the exclusion volume of a Sepharose CL-4B column. Pool 2 was prepared fxom proteoglycans eluting with a mean Kav-value of 0.56 (normal individuals) and of 0.47 (patients), respectively, from the same column. The sum of the radioactive glycosaminoglycans isolated from the different pools was taken as $100 \%$.

The ratio of chondroitin 6-sulfate/chondroitin 4-sulfate was 1 in each individual cell Iine.

2. Chondroitin sulfotransferase activity of cultured fibroblasts

\begin{tabular}{lcc}
\hline & pmole/24 h & chondroitin 4-sulfate \\
Enzyme source & and mg cell protein & chondroitin 6-sulfate \\
\hline Patient 1 & 140 & 1.33 \\
Patient 2 & 120 & 1.05 \\
Controls & 130 & 1.11 \\
& 280 & 0.85 \\
& 150 & 1.28 \\
\hline
\end{tabular}

3. Uptaxe and adsorption of [35S]proteoglycans

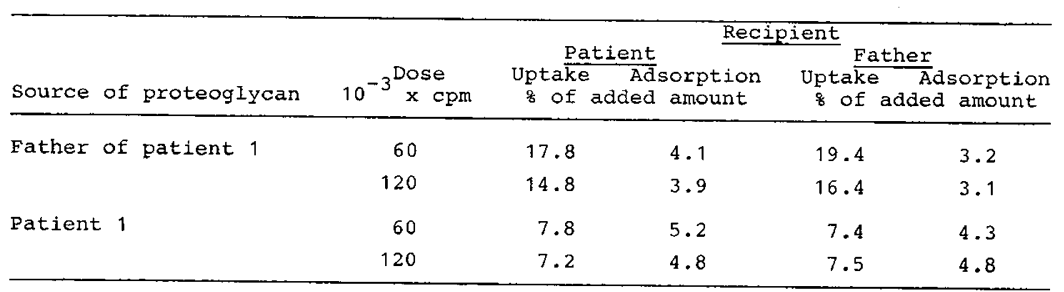

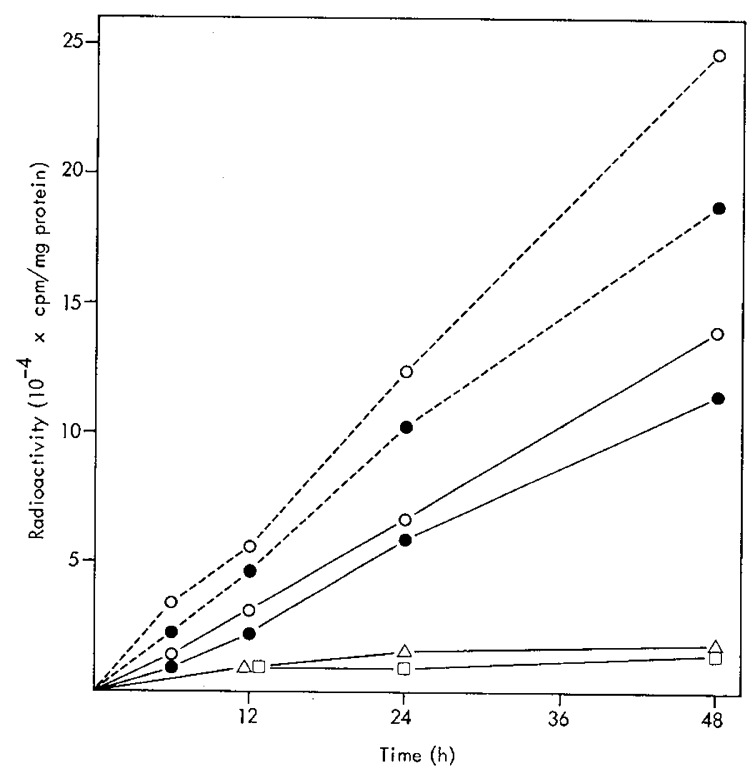

Pigure 1:

Time-dependent incorporation of $\left[{ }^{35} \mathrm{~S}\right]$ sulfate into sulfated glycosaminoglycans.

(D) intracellular and ( ) extracellular glycosaminoglycans of patient $1 ;(\Delta)$ intracellular and $(0)$ extracellular glycosaminoglycans of a normal control; dashed lines represent incorporation in the presence of $1 \mathrm{mM} p$-nitrophenyl- $\beta-D-x y l o s i d e$. 


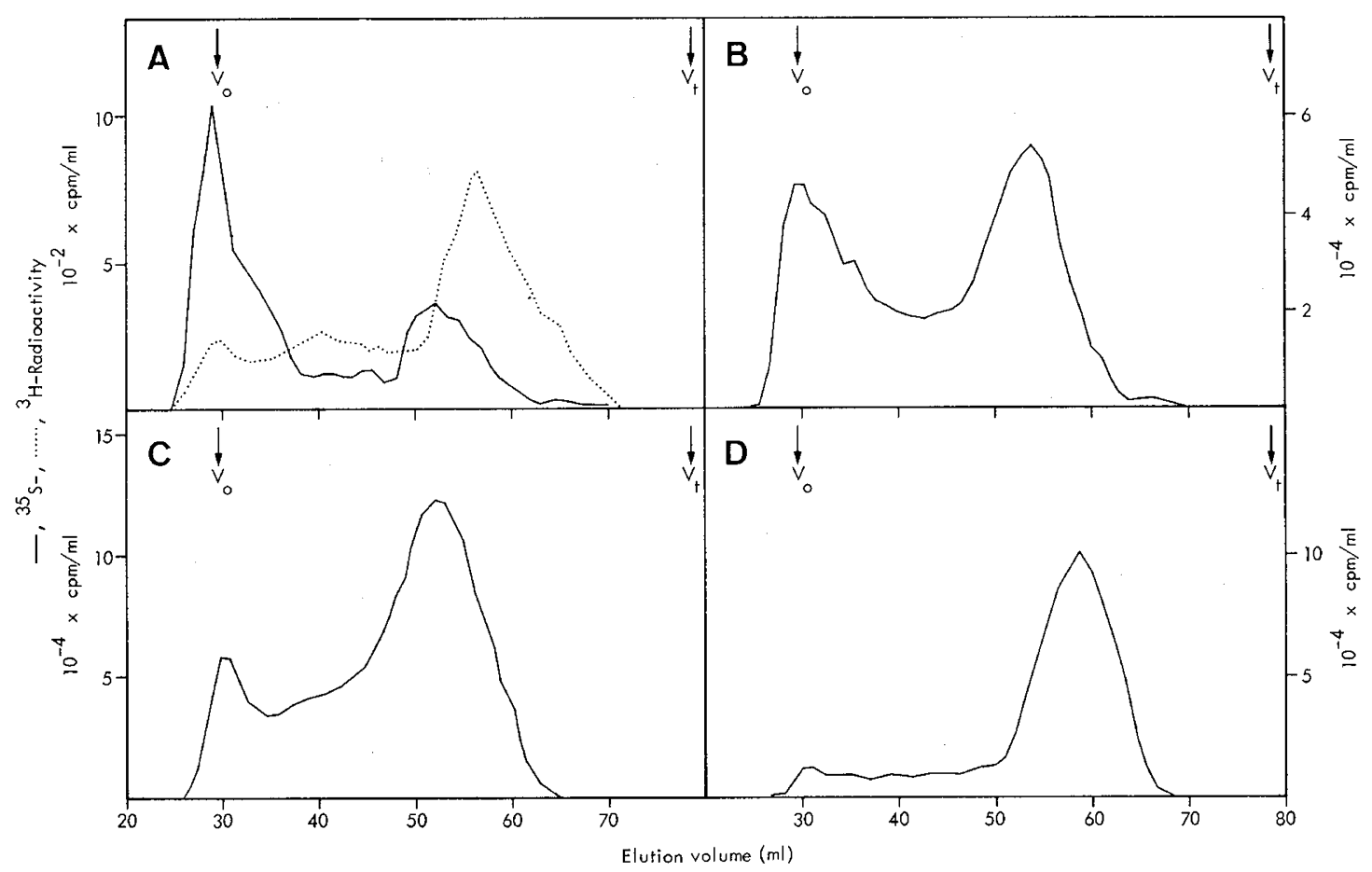

Figure 2:

Gel chromatography on Sepharose CL-4B of $\left[{ }^{35}\right.$ S]proteoglycans

(solid lines) from patient 1 (A), 2 (B) and 3 (C) and from a

normal control (D).

Proteoglycans from patient 1 were cochromatographed with a similar

control.

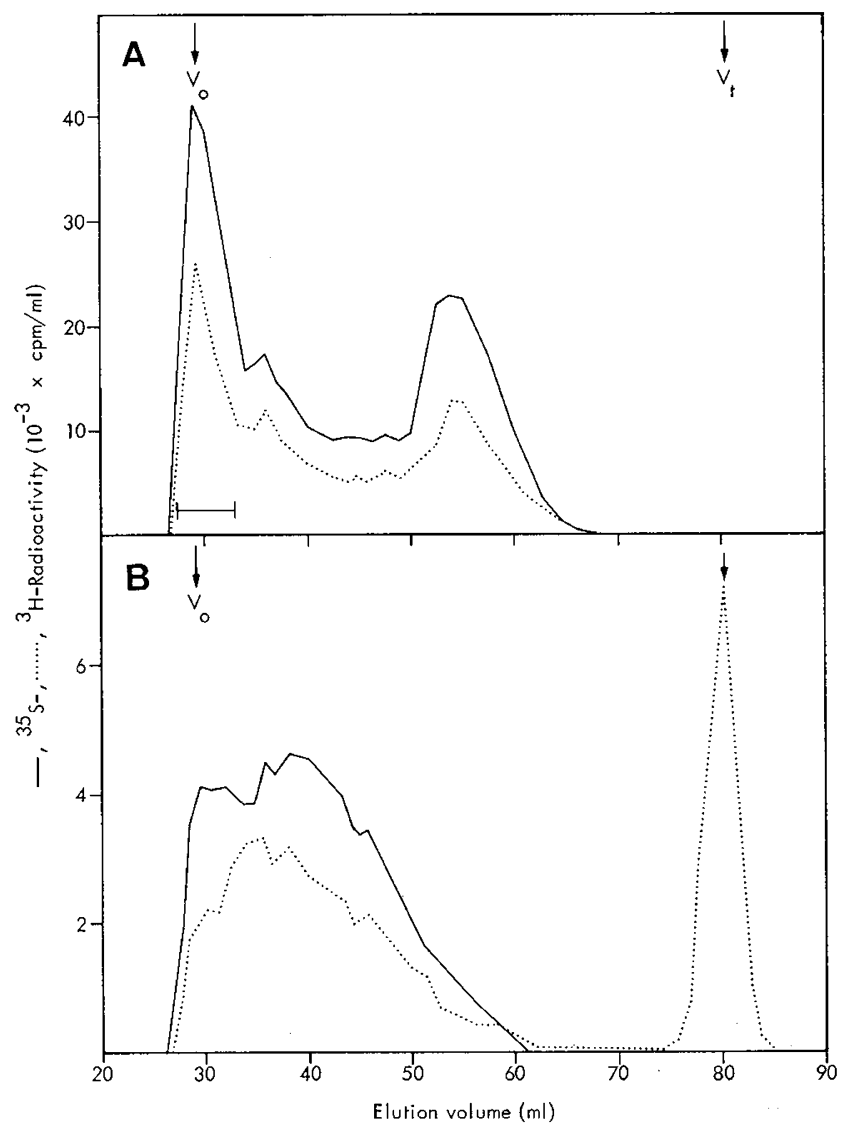

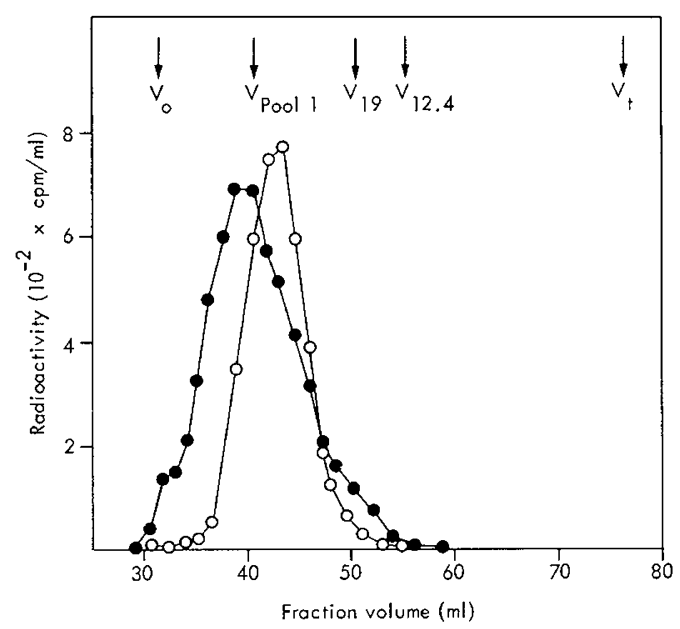

Figure 4:

Gel chromatography on Sephacry 1 s-300 of $\left[{ }^{35}\right.$ S]glycosaminoglycan chains.

(•) pool 2 glycosaminoglycans from patient 1 (those from patient 2 behaved similarly); (0) pool 2 glycosaminoglycans from a normal

control (those from patient 3 behaved similarly). The arrows denote the elution maxima of pool 1 glycosaminoglycans (patients and control) and of glycosaminoglycans with $M_{r}=19,000$ and $M_{r}=12,400$, respectively.

Figure 3:

Gel chromatography on Sepharose $\mathrm{CL}-4 \mathrm{~B}$ of ${ }^{35} \mathrm{~S}-$ and ${ }^{3} \mathrm{H}-\mathrm{labeled}$ proteoglycans from patient 1 (A).

Fractions indicated by the bar were digested with hyaluronate lyase and rechormatographed (B).

Pediat Res. 17: 926-929 (1983) 